\title{
Gender differences in the protective effects of green tea against amnestic mild cognitive impairment in the elderly Han population
}

This article was published in the following Dove Press journal:

Neuropsychiatric Disease and Treatment

\begin{abstract}
Hua Xu, ${ }^{1,2}$ Yaping Wang, ${ }^{3}$ Yefeng Yuan, ${ }^{4}$ Xulai Zhang, ${ }^{5}$ Xiaoyun Zuo, ${ }^{6}$ Lijuan Cui, ${ }^{7}$ Ying Liu, ${ }^{8}$ Wei Chen, ${ }^{9}$ Ning Su, ${ }^{1,2}$ Haihong Wang, ${ }^{1,2}$ Feng Yan, ${ }^{1,2}$ Xia Li, ${ }^{1,2}$ Tao Wang, ${ }^{1,2}$ Shifu Xiao ${ }^{1,2}$

'Department of Geriatric Psychiatry, Shanghai Mental Health Center, Shanghai Jiao Tong University School of Medicine, Shanghai, China; ${ }^{2}$ Alzheimer's Disease and Related Disorders Center, Shanghai Jiao Tong University, Shanghai, China; ${ }^{3}$ Department of Psychology and Psychiatry, The Second Affiliated Hospital of Xi'an Jiao Tong University School of Medicine, Xi'an, Shaanxi Province, China; ${ }^{4}$ Department of Psychiatry, The First Affiliated Hospital of Nanchang University, Nanchang, Jiangxi Province, China; ${ }^{5}$ Department of Geriatric Psychiatry, The Fourth People's Hospital of Hefei City, Hefei, Anhui Province, China; ${ }^{6}$ Department of Geriatrics, The Third People's Hospital of Jian City, Jian, Jiangxi Province, China; ${ }^{7}$ Department of Applied Psychology, East China Normal University, Shanghai, China; ${ }^{8}$ Department of Psychiatry, The First Affiliated Hospital of the China Medical University, Shenyang, Liaoning Province, China; ${ }^{9}$ Department of Psychiatry, Sir Run Run Shaw Hospital, Zhejiang University School of Medicine, Hangzhou, Zhejiang Province, China
\end{abstract}

Correspondence: Shifu Xiao; Tao Wang Alzheimer's Disease and Related Disorders Center, Shanghai Mental Health Center, Shanghai Jiao Tong University School of Medicine, 600 South Wan Ping Road, 200030 Shanghai, China

Tel +8621 64387250

Email xiaoshifu@msn.com; wtshhwy@I63.com
Background: Gender differences may contribute to variances in the potential protective effects of tea against cognitive impairment in the elderly.

Objective: To examine the association between different types of tea consumption and the risk of amnestic mild cognitive impairment (aMCI) along gender lines.

Methods: A cross-sectional study was conducted with reference to 20 communities in China. The sample population included elderly participants aged 60 years or older. A standardized questionnaire was used to collect each participant's general demographic information. Trained psychologists administrated the Mini-Mental State Examination (MMSE) and the Montreal Cognitive Assessment (MoCA) to assess participants' cognitive function. An attending psychiatrist evaluated each participant's cognitive function. Finally, data from 2,131 participants were analyzed to assess the association.

Results: With regard to male participants, the percentage of green tea consumption was higher in the normal control group than in the aMCI group $\left(X^{2}=4.64, P=0.031\right)$. Logistic regression analysis showed that green tea consumption reduced the risk of aMCI in male participants $(\mathrm{OR}=0.657, P=0.019)$, and this finding was highly significant in males aged under 70 years $(\mathrm{OR}=0.376, P=0.002)$. Regarding female participants across every age group, the results indicated that tea consumption failed to significantly decrease the risk of aMCI $(P>0.05)$. Unlike green tea, black tea and oolong tea were not correlated with a reduced risk of aMCI in terms of gender or age group. Multiple linear regression analysis also revealed that age, years of education, and green tea consumption $(B=0.996, P=0.000)$ were associated with MoCA and MMSE scores, though only in male participants.

Conclusion: Green tea consumption showed a protective effect against aMCI in males but not in females, particularly in males aged $<70$ years. However, black tea and oolong tea failed to show any protective effect in either males or females.

Keywords: gender difference, green tea, amnestic mild cognitive impairment, protective factor

\section{Introduction}

Alzheimer's disease (AD) is a progressive neurodegenerative disease that occurs in elderly individuals. AD has a high prevalence and affects people worldwide. Recent studies have indicated that diabetes, midlife hypertension, midlife obesity, physical inactivity, depression, smoking, and low educational attainment are the risk factors for $\mathrm{AD}$, and the evidence is consistent. ${ }^{1}$ Due to the limited treatments available for $\mathrm{AD}$ at this time, emphasis has been placed on the primary and secondary prevention of the disease through the modification of risk factors. ${ }^{2}$ 
Throughout the past decades, the concept of amnestic mild cognitive impairment (aMCI) has emerged in relation to the early detection of AD. Studies have shown that aMCI represents a transitional state between normal aging and $\mathrm{AD} .^{3}$ Although wide variations have been reported in the conversion rate from aMCI to $\mathrm{AD}, \mathrm{aMCI}$ is generally regarded to include the prodromal $\mathrm{AD}$ stage, and the conversion rate of aMCI to $\mathrm{AD}$ is much higher than that of the normal age-matched population. ${ }^{4}$ Among currently identified potential protective or risk factors for cognitive impairment, tea consumption has been regarded to serve a neuroprotective function in the elderly.

Tea drinking was originated in Asia and has since spread throughout the world today. According to the different degrees of fermentation that occur during the manufacturing process, tea can be categorized into three basic types: black tea (fully fermented), oolong tea (semi-fermented), and green tea (nonfermented). Black tea accounts for $\sim 78 \%$ of total tea production worldwide, ${ }^{5}$ and green tea is the most popular type of tea consumed in Asia. Recent studies have suggested that tea drinking is associated with a lower risk of $\mathrm{AD}$ and cognitive impairment. ${ }^{6-9}$ This may be attributed to the effect of catechins, an active ingredient that is especially abundant in green tea. ${ }^{10,11}$ However, the protective effects of tea remain controversial. ${ }^{12}$ Furthermore, the association between the different types of tea consumption and the risk of cognitive impairment have not yet been clarified in previous research studies.

More recently, the researchers of our previous study examined both the protective and risk factors for aMCI in the Shanghai population. The findings revealed different associative effects according to gender and age differences. For example, females were found to be at a higher risk for aMCI than males. Drinking tea was found to be a protective factor for males aged 60 years or older, though this protective effect was not evident in males aged over 70 years. ${ }^{13}$ Due to the limitations of the sample size, the researchers did not analyze the association between different types of tea and cognitive function with regard to more specific age groups or gender differences. Thus, the current study aimed to elaborate upon the association between different types of tea consumption and cognitive performance by recruiting participants from several cities in China in order to obtain a larger sample size for data analysis. This sample here represents a subsample of the China Longitudinal Aging Study (CLAS), and data were analyzed to examine the association observed in males and females.

\section{Methods}

\section{Participants}

The CLAS represented a survey of community-based epidemiological studies in China. ${ }^{14}$ Twenty target communities (ie, 18 urban and 2 rural) located in the eastern, mid, and western parts of China have been included in this study. According to the 2010 national census, permanent residents aged 60 years or older were entered into a database. A simple random sample comprising 4,411 residents was selected to identify potential participants. A total of 3,514 participants completed the baseline survey. Data from the baseline survey were obtained from among the 20 target communities between March 2011 and July 2012 in Han population. Among these participants, a subsample of 2,131 participants included each participant's diagnosis and complete information concerning tea consumption was utilized in our report here. The CLAS project was approved by the Ethics Committee of Shanghai Mental Health Center. All the subjects provided written informed consent for the study before it was initiated.

\section{Neuropsychological tests and tea consumption investigation}

All participants underwent a screening process that consisted in a review of demographic information (ie, name, gender, ethnicity, years of education, and occupation before retirement), information about daily living (ie, hobbies, dietary preferences, smoking history, consumption of alcohol and tea, and physical activities), and type of tea consumed (ie, green tea, black tea, and oolong tea) with frequency of tea consumption.

Psychologists, all of whom had met the same training criteria and who had undergone a consistency evaluation, administered the standardized neuropsychological examinations including the Mini-Mental State Examination (MMSE) ${ }^{15}$ and the Montreal Cognitive Assessment (MoCA).$^{16}$ In addition, the Geriatric Depression Scale ${ }^{17}$ was used to identify depressive symptoms and severity in participants.

\section{Diagnostic criteria}

Attending psychiatrists were assigned the task of collecting information pertaining to medical history, performing physical examinations, and conducting the final assessment. The Activities of Daily Living Scale ${ }^{18}$ the Global Deterioration Scale, ${ }^{19}$ and the Hachinski Ischemia Scale ${ }^{20}$ were used to assist in formulating each diagnosis. The results obtained from MRI scans were also evaluated in order to 
exclude cerebrovascular disease. The diagnosis of aMCI was adapted from the MCI diagnostic criteria reported by Petersen: 1) memory complaints, preferably corroborated by a spouse or relative; 2) objective memory impairment; 3) normal general cognitive function; 4) intact activities of daily living; and 5) absence of dementia. ${ }^{21}$

\section{Data analyses}

All statistical analyses were conducted using IBM SPSS for Windows, version 19 (IBM, Armonk, NY, USA). Independent $t$-tests for the continuous variables were analyzed in order to assess differences in characteristics according to the diagnoses between the gender groups, and chi-square tests were carried out for categorical variables. The independent effect of green tea, black tea, and oolong tea for aMCI was determined using logistic regression analysis, which is adjusted for educational levels for each gender. Finally, multiple linear regressions were carried out to analyze the correlation between the potential protective or risk factors and cognitive function. For all analyses, the significance level was $P<0.05$.

\section{Results}

For both males and females, the average age of the aMCI group was higher than in the normal control (NC) group ( $T=5.15, P=0.000 ; T=8.32, P=0.000$ ), and years of education were higher in the $\mathrm{NC}$ group than in the aMCI group $(T=3.89$, $P=0.000 ; T=12.14, P=0.000 ;$ Table 1$)$. With regard to males, the percentage of green tea consumption was higher in the NC population than in aMCI participants $\left(X^{2}=4.64, P=0.031\right)$. However, black tea consumption; oolong tea consumption; smoking history; drinking history; and history of hypertension, heart disease, endocrine, and metabolic disease did not prove significant when comparisons were made between the aMCI and NC groups $(P>0.05)$.

Logistic regression analysis revealed that the risk of aMCI was lower in male participants who reported green tea consumption ( $\mathrm{OR}=0.657, P=0.019)$. When further analyzed by age, the risk of aMCI was significantly lower in males under 70 years, who had a history of green tea consumption $(\mathrm{OR}=0.376, P=0.002)$. Furthermore, green tea consumption did not prove statistically significant in males over 70 years $(P>0.05)$. In contrast, green tea consumption did not diminish the risk of aMCI in females, regardless of age. Across every age group, neither black tea nor oolong tea was found to be associated with a decreased risk of aMCI $(P>0.05$; Table 2$)$.

To conduct an analysis relating to males and females, multiple linear regression showed that age $(B=-0.221$, $P=0.000)$, years of education $(B=0.309, P=0.000)$, and green tea consumption $(B=0.996, P=0.000)$ were associated with MoCA scores in males, and age $(B=-0.146, P=0.000)$, years of education $(B=0.187, P=0.000)$, and green tea consumption ( $B=0.742, P=0.000$ ) were also associated with the MMSE scores of male participants. Green tea consumption by females was associated with neither MoCA nor MMSE scores $(P>0.05$; Table 3$)$.

Table I Characteristics of the participants in aMCl group and $\mathrm{NC}$ group based on gender

\begin{tabular}{|c|c|c|c|c|c|c|c|c|}
\hline \multirow[t]{2}{*}{ Characteristics } & \multicolumn{4}{|l|}{ Male $(n=965)$} & \multicolumn{4}{|l|}{ Female $(n=I, 166)$} \\
\hline & $\mathrm{aMCl}(\mathrm{n}=\mid \mathbf{5 5})$ & $N C(n=8 \mid 0)$ & $t$ or $X^{2}$ & $P$-value & $\mathrm{aMCl}(\mathrm{n}=284)$ & $N C(n=882)$ & $t$ or $X^{2}$ & $P$-value \\
\hline Age (years) & $73.88 \pm 7.68$ & $70.32 \pm 7.60$ & 5.15 & $0.000 *$ & $74.55 \pm 8.60(n=272)$ & $69.74 \pm 7.36(n=873)$ & 8.32 & $0.000^{*}$ \\
\hline Education (years) & $7.76 \pm 4.97$ & $9.98 \pm 6.35$ & 3.89 & $0.000^{*}$ & 4. $12 \pm 4.21 \quad(n=253)$ & $7.98 \pm 5.07(n=840)$ & 12.14 & $0.000 *$ \\
\hline Tea consumption (\%) & $48.4(n=75)$ & $62.1 \quad(n=504)$ & 10.26 & $0.001 *$ & $21.1(n=60)$ & $26.8(n=236)$ & 3.632 & 0.057 \\
\hline Green tea habit (\%) & $44.5(n=69)$ & $53.9(n=437)$ & 4.64 & $0.03 I^{*}$ & $|8.3|(n=52)$ & $21.09(n=186)$ & 1.02 & 0.312 \\
\hline Black tea habit (\%) & $6.5(n=10)$ & $7.7(n=62)$ & 0.27 & 0.602 & $2.82(n=8)$ & $5.22(n=46)$ & 2.80 & 0.094 \\
\hline Oolong tea habit (\%) & $2.96(n=2)$ & $1.29(n=24)$ & 0.82 & 0.364 & $0.7(n=2)$ & $1.25(n=I I)$ & 0.19 & 0.665 \\
\hline Smoking history (\%) & $51.6(n=80)$ & $54.0(n=437)$ & 0.29 & 0.593 & $6.3(n=18)$ & $4.6(n=40)$ & 1.45 & 0.229 \\
\hline Drinking history (\%) & $41.2(n=63)$ & $37.4(n=302)$ & 0.77 & 0.380 & $4.0(n=I I)$ & $4.0(n=35)$ & 0.00 & 0.983 \\
\hline Hypertension history (\%) & $38.1(n=59)$ & $45.4(n=368)$ & 2.863 & 0.091 & $45.8(n=130)$ & $46.9(n=4 \mid 4)$ & 0.12 & 0.732 \\
\hline Heart disease history (\%) & $16.1 \quad(n=25)$ & $18.3(n=\mid 48)$ & 0.406 & 0.524 & $22.9(n=65)$ & $26.0(n=229)$ & 1.08 & 0.299 \\
\hline $\begin{array}{l}\text { Endocrine and metabolic } \\
\text { disease history (\%) }\end{array}$ & $18.7(n=29)$ & $20.4(n=165)$ & 0.223 & 0.636 & $23.2(n=66)$ & $29.0(n=256)$ & 3.60 & 0.058 \\
\hline MMSE & $24.49 \pm 4.70(n=153)$ & $27.33 \pm 2.86(n=8 \mid 0)$ & 7.22 & $0.000 *$ & $21.32 \pm 5.97(n=284)$ & $26.40 \pm 3.91 \quad(n=880)$ & 13.43 & $0.000^{*}$ \\
\hline MoCA & $19.34 \pm 5.75(n=154)$ & $23.69 \pm 4.40(n=804)$ & 8.91 & $0.000 *$ & $15.30 \pm 5.98(n=282)$ & $22.17 \pm 5.69(n=878)$ & 17.42 & $0.000 *$ \\
\hline
\end{tabular}

Notes: $P$-value was calculated from independent $t$-test for continuous variables and chi-square test for dichotomous variables; $* P<0.05$; tea habit means at least three cases of tea drinking a week. Drinking history means an average alcohol consumption of $>20 \mathrm{~g} /$ day.

Abbreviations: aMCl, amnestic mild cognitive impairment; MMSE, Mini-Mental State Examination; MoCA, Montreal Cognitive Assessment; NC, normal control. 
Table 2 The protective effects of three types of tea from aMCl in gender- and age-specific groups

\begin{tabular}{|c|c|c|c|c|c|c|c|c|c|}
\hline & \multicolumn{3}{|c|}{ Green tea } & \multicolumn{3}{|c|}{ Black tea } & \multicolumn{3}{|c|}{ Oolong tea } \\
\hline & OR & $95 \% \mathrm{Cl}$ & $P$-value & OR & $95 \% \mathrm{Cl}$ & $P$-value & OR & $95 \% \mathrm{Cl}$ & $P$-value \\
\hline All male $(n=965)$ & 0.657 & $0.46-0.93$ & $0.019 *$ & 0.738 & $0.37-1.49$ & 0.397 & 0.390 & $0.09-1.68$ & 0.206 \\
\hline $55-69$ years $(n=473)$ & 0.376 & $0.20-0.70$ & $0.002 *$ & 0.174 & $0.02-|.3|$ & 0.090 & 0.000 & 0.000 & 0.999 \\
\hline $70-79$ years $(n=360)$ & 0.802 & $0.64-1.79$ & 0.802 & 0.193 & $0.74-4.36$ & 0.193 & 0.934 & $0.22-5.31$ & 0.934 \\
\hline 80 years and more $(n=\mid 32)$ & 0.652 & $0.28-1.51$ & 0.318 & 0.279 & $0.03-2.37$ & 0.242 & 0.000 & 0.000 & 0.999 \\
\hline All female $(n=I, I 66)$ & 0.82 & $0.58-1.16$ & 0.261 & 0.52 & $0.24-1.12$ & 0.093 & 0.60 & $0.13-2.72$ & 0.504 \\
\hline $55-69$ years $(n=58 I)$ & 1.06 & $0.62-1.80$ & 0.840 & 0.35 & $0.08-1.48$ & 0.152 & 0.99 & $0.11-8.58$ & 0.992 \\
\hline $70-79$ years $(n=396)$ & 0.96 & $0.56-1.65$ & 0.890 & 0.65 & $0.18-2.36$ & 0.512 & 0.49 & $0.06-4.18$ & 0.513 \\
\hline 80 years and more $(n=189)$ & 0.43 & $0.18-1.03$ & 0.057 & 0.84 & $0.18-3.90$ & 0.828 & None & None & None \\
\hline
\end{tabular}

Notes: Multiple logistic regression was used to estimate the $\mathrm{OR}$ and $95 \% \mathrm{Cl}$ of tea consumption and the risk of aMCl, adjusted for educational levels; none indicates no participants; aged $>80$ years had the habit of oolong tea consumption; $* P<0.05$.

Abbreviation: $\mathrm{aMCl}$, amnestic mild cognitive impairment.

\section{Discussion}

The present study utilized a subsample of cross-sectional data derived from the CLAS project involving the Chinese population, and this may be the first study to report the association between different types of tea consumption and cognitive function according to the gender differences. The results revealed the following: 1) For both males and females, younger age and higher education levels were found to be protective factors against aMCI during old age, yet only in men did tea consumption appear to elicit protective effects from aMCI. 2) Among the different types of tea, only green tea showed protective effects against cognitive impairment. Neither black tea nor oolong tea elicited protective effects. Furthermore, this protective effect proved highly significant in male participants aged under 70 years, but not in participants aged over 70 years. 3) The scores of male participants obtained from cognitive function scale assessments (MoCA and MMSE) were associated with age, education level, and green tea drinking, but the scores of the cognitive evaluation of female participants were only associated with age and education. No correlation was found between tea consumption and cognitive function in female participants.

The present study offers further support for a growing body of evidence that suggests that the age and level of education are significant factors that influence the risk of aMCI. ${ }^{22,23}$ In contrast to these aforementioned factors, the association between tea consumption and the risk of aMCI currently remains unclear. The results of this study showed tea consumption to be a protective factor against aMCI in older males, though subsequent analysis according to different types of tea failed to offer any evidence in support of the protective effect of black or oolong tea in either age- or gender-specific group. The majority of previous studies have neglected to stratify tea by type. Indeed, some studies have reported that tea, in general, whether green, black, or oolong tea, may serve as a protective factor against cognitive decline

Table 3 The correlative factors of cognitive performance (MoCA and MMSE) for different genders

\begin{tabular}{|c|c|c|c|c|c|c|c|c|}
\hline & \multicolumn{4}{|c|}{ MoCA (male, $n=965)$} & \multicolumn{4}{|c|}{ MoCA (female, $n=I, 166$ ) } \\
\hline & B & SE & Adjusted B & $P$-value & B & SE & Adjusted B & $P$-value \\
\hline Age & -0.221 & 0.018 & -0.344 & $0.000 *$ & -0.260 & 0.018 & -0.315 & $0.000 *$ \\
\hline Education & 0.309 & 0.022 & 0.390 & $0.000 *$ & 0.714 & 0.028 & 0.569 & $0.000 *$ \\
\hline Green tea & 0.996 & 0.279 & 0.101 & $0.000 *$ & 0.155 & 0.327 & 0.010 & 0.635 \\
\hline Black tea & -1.001 & 0.519 & -0.054 & 0.054 & 0.011 & 0.619 & 0.000 & 0.986 \\
\hline \multirow[t]{3}{*}{ Oolong tea } & 0.411 & 0.815 & 0.014 & 0.614 & -0.938 & 1.207 & -0.016 & 0.437 \\
\hline & \multicolumn{4}{|c|}{ MMSE (male, $n=965$ ) } & \multicolumn{4}{|c|}{ MMSE (female, $n=1,166$ ) } \\
\hline & B & SE & Adjusted B & $P$-value & B & SE & Adjusted B & $P$-value \\
\hline Age & -0.146 & 0.013 & -0.336 & $0.000 *$ & -0.192 & 0.015 & -0.302 & $0.000 *$ \\
\hline Education & 0.187 & 0.016 & 0.348 & $0.000 *$ & 0.514 & 0.023 & 0.530 & $0.000 *$ \\
\hline Green tea & 0.742 & 0.196 & 0.110 & $0.000 *$ & 0.054 & 0.27 I & 0.004 & 0.842 \\
\hline Black tea & -0.051 & 0.365 & -0.004 & 0.889 & 0.184 & 0.514 & 0.008 & 0.720 \\
\hline Oolong tea & -0.026 & 0.572 & -0.001 & 0.964 & 0.174 & 1.002 & 0.004 & 0.862 \\
\hline
\end{tabular}

Notes: Multiple linear regressions were used to estimate the correlation between factors and cognitive performance. $* P<0.05$.

Abbreviations: MMSE, mini-mental state examination; MoCA, montreal cognitive assessment; SE, standard error. 
in the elderly. ${ }^{24-26}$ In contrast to a number of such studies, the findings of Noguchi-Shinohara et al concur with the results of our study, suggesting an association between green tea consumption, as opposed to black tea consumption, and a reduced risk of cognitive decline. ${ }^{27}$ These inconsistencies in results may be attributed to a lesser quantity of catechins, which is understood to be associated with the latent mechanisms of the neuroprotective effect of tea contained in black tea and oolong tea as opposed to green tea. The amount of catechins is generally lower in black or oolong tea because of the enzymatic transformation that takes place during fermentation. ${ }^{24,28}$ Thus, these types of tea may fail to elicit a protective effect. However, the rate of black tea or oolong tea consumption was still too low in the sample used in the current study. As such, the influence of these teas on cognitive impairment may be too minute to detect. In light of such inconsistencies in results, the recommended amount of catechins or the other active ingredients associated with protective effects should be further explored.

A large amount of research has cumulatively suggested that green tea may be a protective factor against cognitive dysfunction in the elderly. ${ }^{29}$ Possible mechanisms involved in the protective effects of tea may be attributable to their antioxidant and iron-chelating properties as well as to the modulation of cell signaling and cell survival pathways. ${ }^{30-32}$ However, several other studies have shown that the association between tea consumption and the risk of cognitive impairment was too weak to draw any conclusion. ${ }^{33}$ Our present study explored the association between green tea consumption and cognitive decline according to gender- and age-specific differences. The findings showed green tea to be a protective factor, though only in males. This suggests that females across every age group cannot benefit from tea consumption. Furthermore, the protective effect of green tea in males was deemed highly significant only in participants $<70$ years as old and not in older individuals. Huang interviewed 823 elderly participants in West China aged 90-108 years and found similar results that indicated that the protective effect of green tea varies according to gender. ${ }^{34}$ They found an association between cognitive impairment and tea consumption habits in males. However, no such association was found in females. In support of Huang, the present study found an overall, male-specific significant association between green tea consumption and reduced aMCI risk. However, this finding proved highly significant in participants aged under 70 years. The results also revealed an association between the scores of male participants, as measured utilizing cognitive function scales, and green tea consumption. This finding furthermore supports the idea that green tea is a protective factor for male cognitive function. It is widely accepted that cognitive impairment is a complex process of multiple-factor interactions. Age, sex, educational level, genetic predispositions, lifestyle, and physical disease may contribute to the dynamic process of cognitive function impairment. ${ }^{35}$ The findings of the current study suggest that authentic green tea consumption may contribute to protecting cognitive function in the elderly that such consumption may be more effective for males at an earlier stage in their life span and green tea consumption may be considered in terms of the strategies employed in the primary prevention of dementia.

There is a growing awareness of gender differences in epidemiology as well as in the clinical manifestation and treatment strategy in dementia. ${ }^{36-43}$ It is currently understood that two-thirds of individuals with AD are female. Although some of this difference is attributable to the fact that women live longer, ${ }^{44}$ this is still not sufficient to explain the fact that women are 1.5 times more likely than men to develop AD. ${ }^{22}$ Additional evidence derives from the APOEE4 allele, which is known to increase the risk of dementia and which may be mediated by estrogen in women. ${ }^{42,45}$ Research evidence has also shown that brain structure and function in men and women differ throughout the aging process. ${ }^{46}$ In general, different genetics, hormones, metabolisms, brain aging processes, and stress coping mechanisms are detectable between the genders. ${ }^{36,38,41}$ The findings of the current study suggest that gender differences exert an influence on the protective effects of green tea, revealing that such effects are detectable only in males. Although the cause of the only protective effect in males remains elusive, it nonetheless highlights the need to consider protective and risk factors in men and women independently, which may serve to accelerate etiological research as well as the implementation of differential preventive measures.

The strengths of the present study are illustrated in the utilization of gender- and age-specific groups to examine cognitive function in the elderly in China. The researchers found that green tea may be a protective factor against cognitive decline in males as opposed to females. Furthermore, the protective effect proved significant in males $<70$ years of age. Thus, the findings offer support for the hypothesis that gender differences may significantly influence the onset of dementia. The present study has several limitations. First, the study failed to elaborate upon the association between green tea consumption and cognitive function by engaging in a precise quantitative analysis of the amount of tea and 
the risk of cognitive decline. Second, the current research is a cross-sectional study and cannot identify a direct causal link between green tea consumption and cognitive decline. Therefore, the conclusions outlined in this study require validation through further longitudinal studies. Finally, the rate of oolong tea consumption proved too low to determine a correlation with the risk of aMCI.

\section{Conclusion}

The present study found gender to exert an influence on the association between green tea consumption and the risk of cognitive impairment. In contrast to female participants, elderly male participants were shown to benefit from green tea consumption, as opposed to black tea or oolong tea consumed prior to reaching 70 years. As the current research constitutes a cross-sectional study, further longitudinal studies are required to validate the findings. In conclusion, this study supports the hypothesis that gender difference may significantly influence the risk of cognitive decline in the elderly, a finding which should be considered in terms of the primary prevention of dementia.

\section{Acknowledgments}

This work was supported by grants from the China Ministry of Science and Technology (2009BAI77B03), Shanghai Jiao Tong University School of Medicine (15ZH4010), and Shanghai Jiaotong University School of Medicine and Institute of neuroscience, Chinese Academy of Sciences (2015NKX003).

\section{Disclosure}

The authors report no conflicts of interest in this work.

\section{References}

1. Williams JW, Plassman BL, Burke J, Benjamin S. Preventing Alzheimer's disease and cognitive decline. Evid Rep Technol Assess (Full Rep). 2010; 193:1-727.

2. Imtiaz B, Tolppanen AM, Kivipelto M, Soininen H. Future directions in Alzheimer's disease from risk factors to prevention. Biochem Pharmacol. 2014;88(4):661-670.

3. Petersen RC, Smith GE, Waring SC, Ivnik RJ, Tangalos EG, Kokmen E. Mild cognitive impairment: clinical characterization and outcome. Arch Neurol. 1999;56(3):303-308.

4. Petersen RC, Aisen PS, Beckett LA, et al. Alzheimer's Disease Neuroimaging Initiative (ADNI): clinical characterization. Neurology. 2010; 74(3):201-209.

5. Cheng TO. All teas are not created equal: the Chinese green tea and cardiovascular health. Int J Cardiol. 2006;108(3):301-308.

6. Yang L, Jin X, Yan J, et al. Prevalence of dementia, cognitive status and associated risk factors among elderly of Zhejiang province, China in 2014. Age Ageing. 2016;45(5):708-712.

7. Tomata Y, Sugiyama K, Kaiho Y, et al. Green tea consumption and the risk of incident dementia in elderly Japanese: the Ohsaki Cohort 2006 Study. Am J Geriatr Psychiatry. 2016;24(10):881-889.
8. Ide K, Yamada H, Takuma N, et al. Green tea consumption affects cognitive dysfunction in the elderly: a pilot study. Nutrients. 2014;6(10): 4032-4042.

9. Kuriyama S, Hozawa A, Ohmori K, et al. Green tea consumption and cognitive function: a cross-sectional study from the Tsurugaya Project 1. Am J Clin Nutr. 2006;83(2):355-361.

10. Choi YT, Jung CH, Lee SR, et al. The green tea polyphenol (-)-epigallocatechin gallate attenuates beta-amyloid-induced neurotoxicity in cultured hippocampal neurons. Life Sci. 2001;70(5):603-614.

11. Xicota L, Rodriguez-Morato J, Dierssen M, de la Torre R. Potential role of (-)-Epigallocatechin-3-Gallate (EGCG) in the secondary prevention of Alzheimer disease. Curr Drug Targets. 2017;18(2):174-195.

12. Lindsay J, Laurin D, Verreault R, et al. Risk factors for Alzheimer's disease: a prospective analysis from the Canadian Study of Health and Aging. Am J Epidemiol. 2002;156(5):445-453.

13. Wang T, Xiao S, Chen K, et al. Prevalence, incidence, risk and protective factors of amnestic mild cognitive impairment in the elderly in Shanghai. Curr Alzheimer Res. 2017;14(4):460-466.

14. Xiao S, Li J, Tang M, et al. Methodology of China's national study on the evaluation, early recognition, and treatment of psychological problems in the elderly: the China Longitudinal Aging Study (CLAS). Shanghai Arch Psychiatry. 2013;25(2):91-98.

15. Folstein MF, Folstein SE, McHugh PR. "Mini-mental state": a practical method for grading the cognitive state of patients for the clinician. J Psychiatr Res. 1975;12(3):189-198.

16. Nasreddine ZS, Phillips NA, Bedirian V, et al. The Montreal Cognitive Assessment, MoCA: a brief screening tool for mild cognitive impairment. J Am Geriatr Soc. 2005;53(4):695-699.

17. Yesavage JA, Brink TL, Rose TL, et al. Development and validation of a geriatric depression screening scale: a preliminary report. J Psychiatr Res. 1982;17(1):37-49.

18. Lawton MP, Brody EM. Assessment of older people: self-maintaining and instrumental activities of daily living. Gerontologist. 1969;9(3): 179-186.

19. Reisberg B, Ferris SH, de Leon MJ, Crook T. The Global Deterioration Scale for assessment of primary degenerative dementia. Am J Psychiatry. 1982;139(9):1136-1139.

20. Hachinski VC, Iliff LD, Zilhka E, et al. Cerebral blood flow in dementia. Arch Neurol. 1975;32(9):632-637.

21. Petersen RC, Doody R, Kurz A, et al. Current concepts in mild cognitive impairment. Arch Neurol. 2001;58(12):1985-1992.

22. Gao S, Hendrie HC, Hall KS, Hui S. The relationships between age, sex, and the incidence of dementia and Alzheimer disease: a meta-analysis. Arch Gen Psychiatry. 1998;55(9):809-815.

23. Povova J, Ambroz P, Bar M, et al. Epidemiological of and risk factors for Alzheimer's disease: a review. Biomed Pap Med Fac Univ Palacky Olomouc Czech Repub. 2012;156(2):108-114.

24. Luczaj W, Skrzydlewska E. Antioxidative properties of black tea. Prev Med. 2005;40(6):910-918.

25. Bastianetto S, Yao ZX, Papadopoulos V, Quirion R. Neuroprotective effects of green and black teas and their catechin gallate esters against beta-amyloid-induced toxicity. Eur J Neurosci. 2006;23(1):55-64.

26. Grelle G, Otto A, Lorenz M, Frank RF, Wanker EE, Bieschke J. Black tea theaflavins inhibit formation of toxic amyloid-beta and alphasynuclein fibrils. Biochemistry. 2011;50(49):10624-10636.

27. Noguchi-Shinohara M, Yuki S, Dohmoto C, et al. Consumption of green tea, but not black tea or coffee, is associated with reduced risk of cognitive decline. PLoS One. 2014;9(5):e96013.

28. Henning SM, Niu Y, Lee NH, et al. Bioavailability and antioxidant activity of tea flavanols after consumption of green tea, black tea, or a green tea extract supplement. Am J Clin Nutr. 2004;80(6):1558-1564.

29. Ng TP, Feng L, Niti M, Kua EH, Yap KB. Tea consumption and cognitive impairment and decline in older Chinese adults. Am J Clin Nutr. 2008; 88(1):224-231.

30. Weinreb O, Mandel S, Amit T, Youdim MB. Neurological mechanisms of green tea polyphenols in Alzheimer's and Parkinson's diseases. J Nutr Biochem. 2004;15(9):506-516. 
31. Mandel S, Youdim MB. Catechin polyphenols: neurodegeneration and neuroprotection in neurodegenerative diseases. Free Radic Biol Med. 2004;37(3):304-317.

32. Hou RR, Chen JZ, Chen H, Kang XG, Li MG, Wang BR. Neuroprotective effects of (-)-epigallocatechin-3-gallate (EGCG) on paraquatinduced apoptosis in PC12 cells. Cell Biol Int. 2008;32(1):22-30.

33. Panza F, Solfrizzi V, Barulli MR, et al. Coffee, tea, and caffeine consumption and prevention of late-life cognitive decline and dementia: a systematic review. J Nutr Health Aging. 2015;19(3):313-328.

34. Huang CQ, Dong BR, Zhang YL, Wu HM, Liu QX. Association of cognitive impairment with smoking, alcohol consumption, tea consumption, and exercise among Chinese nonagenarians/centenarians. Cogn Behav Neurol. 2009;22(3):190-196.

35. Norton S, Matthews FE, Barnes DE, Yaffe K, Brayne C. Potential for primary prevention of Alzheimer's disease: an analysis of populationbased data. Lancet Neurol. 2014;13(8):788-794.

36. Carter CL, Resnick EM, Mallampalli M, Kalbarczyk A. Sex and gender differences in Alzheimer's disease: recommendations for future research. J Womens Health (Larchmt). 2012;21(10):1018-1023.

37. Ordonez C, Navarro A, Perez C, Martínez E, del Valle E, Tolivia J. Gender differences in apolipoprotein $\mathrm{D}$ expression during aging and in Alzheimer disease. Neurobiol Aging. 2012;33(2):433.e11-e20.

38. Li R, Singh M. Sex differences in cognitive impairment and Alzheimer's disease. Front Neuroendocrinol. 2014;35(3):385-403.
39. Mielke MM, Vemuri P, Rocca WA. Clinical epidemiology of Alzheimer's disease: assessing sex and gender differences. Clin Epidemiol. 2014;6:37-48.

40. Rocca WA, Mielke MM, Vemuri P, Miller VM. Sex and gender differences in the causes of dementia: a narrative review. Maturitas. 2014 79(2):196-201.

41. Austad SN, Bartke A. Sex differences in longevity and in responses to antiaging interventions: a mini-review. Gerontology. 2015;62(1):40-46.

42. Xing Y, Tang Y, Jia J. Sex differences in neuropsychiatric symptoms of Alzheimer's disease: the modifying effect of apolipoprotein E $\varepsilon 4$ status. Behav Neurol. 2015;2015:275256.

43. Podcasy JL, Epperson CN. Considering sex and gender in Alzheimer disease and other dementias. Dialogues Clin Neurosci. 2016;18(4): $437-446$.

44. Bennett DA. Editorial comment on "Prevalence of dementia in the United States: the aging, demographics, and memory study" by Plassman et al. Neuroepidemiology. 2007;29(1-2):133-135.

45. Altmann A, Tian L, Henderson VW, Greicius MD; Alzheimer's Disease Neuroimaging Initiative Investigators. Sex modifies the APOErelated risk of developing Alzheimer disease. Ann Neurol. 2014;75(4): $563-573$.

46. Giedd JN, Raznahan A, Mills KL, Lenroot RK. Review: magnetic resonance imaging of male/female differences in human adolescent brain anatomy. Biol Sex Differ. 2012;3(1):19.

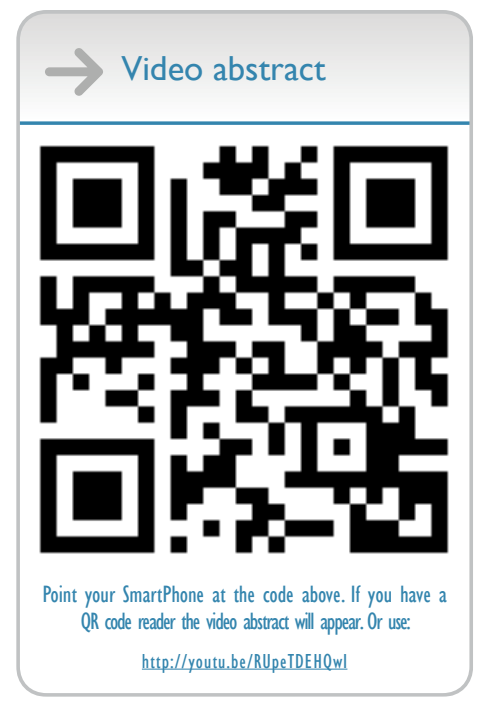

Neuropsychiatric Disease and Treatment

\section{Publish your work in this journal}

Neuropsychiatric Disease and Treatment is an international, peerreviewed journal of clinical therapeutics and pharmacology focusing on concise rapid reporting of clinical or pre-clinical studies on a range of neuropsychiatric and neurological disorders. This journal is indexed on PubMed Central, the 'PsycINFO' database and CAS,

\section{Dovepress}

and is the official journal of The International Neuropsychiatric Association (INA). The manuscript management system is completely online and includes a very quick and fair peer-review system, which is all easy to use. Visit http://www.dovepress.com/testimonials.php to read real quotes from published authors. 